\title{
O trabalho do enfermeiro cirúrgico e o potencial para minimizar complicações pós-operatórias
}

\author{
The work surgical nurse and power to minimized postoperative \\ El trabajo del enfermero quirúrgico y la posibilidad de minimizar las complicaciones \\ postoperatorias
}

\begin{abstract}
Leticia Silveira Cardoso ${ }^{1}$, Joseane Trindade Nogueira ${ }^{1}$, Jamille Louise Bortoni de Oliveira Lopes ${ }^{1}$, Amanda Peres de Souza ${ }^{1}$, Luciana Araujo Vieira², Ana Karina Silva da Rocha Tanaka ${ }^{2,3}$, Rosaura Soares Paczek ${ }^{3}$, Letiére Silveira Cardoso ${ }^{4}$, Aléxia Cardozo Scherer ${ }^{1}$, Letice Dalla Lana ${ }^{1,2 *}$.
\end{abstract}

\section{RESUMO}

Objetivo: Relatar o potencial do trabalho do enfermeiro de centro cirúrgico para minimizar complicações pósoperatórias. Relato de experiência: Construído por discentes e docentes de enfermagem a partir de atividades práticas em um Centro Cirúrgico de um hospital filantrópico. Os resultados foram categorizados em: o potencial para a segurança do paciente está relacionada a utilização de instrumentos como Lista de Verificação de Cirurgia Segura, enquanto que o potencial de autonomia está na implementação de instrumentos que permitam a comunicação verbal e escrita para a continuidade da assistência, bem como gerenciamento do serviço. O potencial para o trabalho colaborativo está na ação conjunta entre os envolvidos nos períodos pré-intra e pós-operatório do paciente, os quais permitem minimizar complicações pósoperatórias. Considerações finais: Fica evidente, a partir da vivência em campo prático, a importância do papel do enfermeiro de centro cirúrgico para minimizar complicações pós-operatórias. As não conformidades identificadas na prática pelos discentes demonstram fragilidades que devem ser reorganizadas para garantir a segurança do paciente, desenvolvimento da autonomia profissional e trabalho colaborativo. Todavia, observa-se que o enfermeiro ao deter-se de conhecimento e instrumentos de avaliação do cuidado, reforçam sua autonomia profissional e trabalho colaborativo.

Palavras-chave: Segurança do paciente, Enfermagem de centro cirúrgico, Cuidados pós-operatórios, Estudantes de enfermagem.

\section{ABSTRACT}

Objective: To report the work potential of nurses in the operating room to minimize postoperative complications. Experience report: Built by nursing students and teachers from practical activities in a Surgical Center of a philanthropic hospital. The results were categorized into: the potential for patient safety is related to the use of instruments such as the Safe Surgery Checklist, while the potential for autonomy lies in the implementation of instruments that provide verbal and written communication for continuity of maintenance, as well as service management. The potential for collaborative work is in the joint action between those involved in the patient's pre-intra and postoperative periods, which allow to minimize postoperative complications. Final considerations: Es evidente, por la experiencia en el área práctica, la importancia del rol del enfermero en el quirófano para minimizar las complicaciones postoperatorias. The non-conformities identified in practice by the students demonstrate weaknesses that must be reorganized to ensure patient safety, development of professional autonomy and collaborative work. However, it is observed that the nurse when determining whether the knowledge and the instruments for evaluating care, reinforce their professional autonomy and collaborative work.

Keywords: Patient safety, Operating room nursing, Postoperative care, Students nursing.

${ }^{1}$ Universidade Federal do Pampa (UNIPAMPA), Uruguaiana - RS. *E-mail: leticedl@hotmail.com

${ }^{2}$ Associação Brasileira de Enfermagem (ABE), Porto Alegre - RS.

${ }^{3}$ Universidade Federal do Rio Grande do Sul (UFRGS), Porto Alegre - RS.

${ }^{4}$ Sistema Nacional de Aprendizagem do Comércio (SENAC), Uruguaiana - RS.

SUBMETIDO EM: 9/2020

ACEITO EM: 10/2020

PUBLICADO EM: 12/2020 
RESUMEN

Objetivo: Informar sobre el potencial de trabajo del enfermero en quirófano para minimizar las complicaciones postoperatorias. Informe de experiencia: Elaborado por estudiantes y profesores de enfermería a partir de actividades prácticas en un Centro Quirúrgico de un hospital filantrópico. Los resultados se clasificaron em: el potencial de la enfermera para la seguridad del paciente quirúrgico, el potencial de la enfermera para el desarrollo de la autonomía profesional y el potencial de la enfermera para el trabajo colaborativo. El potencial para la seguridad del paciente está relacionado con el uso de instrumentos como la Lista de Verificación de Cirugía Segura, mientras que el potencial de autonomía radica en la implementación de instrumentos que brinden comunicación verbal y escrita para la continuidad del mantenimiento, así como la gestión del servicio. El potencial de trabajo colaborativo está en la acción conjunta entre los involucrados en los periodos pre-intra y postoperatorio del paciente, que permitan minimizar las complicaciones postoperatorias. Consideraciones finales: Las no conformidades identificadas en la práctica por los estudiantes evidencian debilidades que deben ser reorganizadas para garantizar la seguridad del paciente, el desarrollo de la autonomía profesional y el trabajo colaborativo. Sin embargo, se observa que el enfermero a la hora de determinar si los conocimientos y los instrumentos para evaluar el cuidado refuerzan su autonomía profesional y trabajo colaborativo.

Palabras clave: Seguridad del paciente, Enfermería de quirófano, Cuidados posoperatorios, Estudiantes de enfermería.

\section{INTRODUÇÃO}

O trabalho do profissional enfermeiro está presente expressivamente em todos os serviços de assistência à saúde. Uma vez que, a atuação da equipe de enfermagem na realização de cuidados a pessoas e famílias deve ocorrer sobre sua supervisão. Na particularidade dos serviços hospitalares de atenção cirúrgica 0 profissional enfermeiro possui diferentes atribuições. Estas podem ser descritas sob duas perspectivas, quais sejam: das ações gerenciais e das ações assistências, às quais se entrelaçam e representam um dos potenciais do trabalho do enfermeiro cirúrgico (COFEN, 2017; CARVALHO R e BIANCHI ERF, 2016).

Cabe destacar que o trabalho do profissional enfermeiro cirúrgico perpassa todos os períodos da enfermagem perioperatório. Tem-se assim seu início no período pré-operatório com a decisão pelo tratamento, ou seja, quando se opta pela realização da intervenção cirúrgica. Tal decisão deve ser compartilhada entre médico, enfermeiro, paciente e familiar, já que toda intervenção cirúrgica apresenta risco a vida e necessita de autorização prévia, exceto em casos de emergência. Neste, não há possibilidade de obter-se o consentimento informado do paciente e nem há responsáveis pelo paciente presente, logo cabe aos profissionais de saúde envolvidos na assistência à saúde cumprirem seus juramentos respaldados nos princípios bioéticos de beneficência, não maleficência e que buscam salvaguardar a vida do paciente e esclarecer os procedimentos que serão realizados autonomia (MACHADO MAG, 2016).

O período pré-operatório não possui um intervalo de tempo igual entre a decisão pelo procedimento cirúrgico e a realização do mesmo para todos os pacientes, os quais os classificam em: cirurgia de emergência, urgência ou eletiva. Este período que determina o tipo de cirurgia tem relação com a presença ou não de complicações intra e/ou pós-operatórias como hemorragia, choque anafilático, tromboembolia, parada cardiorrespiratória. $O$ tempo entre a decisão e a realização do procedimento permitirá uma avaliação laboratorial prévia, investigação e avaliação clínica de fatores de risco, como exemplo a identificação de alergias, presença de vias aéreas difíceis à intubação endotraqueal ou a condição nutricional do paciente, para que se possa obter sucesso na intervenção cirúrgica (DIEGO LAS, et al., 2016; ZAMPER RPC, et al., 2017).

A intervenção cirúrgica inicia com o posicionamento do paciente na mesa operatória, atribuição do profissional enfermeiro no tocante a suas atividades assistências em centro cirúrgico. $O$ posicionamento cirúrgico adequado contribui para que não ocorram eventos adversos durante o período intraoperatório. Entre eles têm-se as complicações cirúrgicas referidas anteriormente, como também as lesões teciduais acidentais tais como as queimaduras térmicas em uso do eletrocautério. 
Todas as complicações produzidas no intraoperatório permaneceram no período denominado como pósoperatório, seja na fase imediata que se inicia com a recepção do paciente na Sala de Recuperação PósAnestésica (SRPA) e finda após 48 horas do início da intervenção anestésico-cirúrgica ou na fase mediata. Esta perdura de 5 a 7 dias após o término da imediata e, em alguns casos tem-se ainda as complicações pósoperatórias tardias que se estendem até um ano após a cirurgia (MARTINS FZ e DALL'AGNOL CM, 2016; CARVALHO R, BIANCHI ERF, 2016; SOBRAL GAS, et al., 2019).

Frente ao longo tempo possível para a ocorrência de complicações pós-operatórias, bem como pelas elevadas taxas de infecção do sítio cirúrgico apontadas em estudos nacionais e internacionais, justifica-se enfatizar o potencial existente no trabalho do enfermeiro cirúrgico para minimizá-las ou mesmos evitá-las. A isto acresce-se ao expressivo custo financeiro disponibilizado para as reintervenções cirúrgicas, para o uso de antibioticoterapia e para manutenção dos dias de internação hospitalar dos pacientes que apresentam complicações pós-operatórias.

E, por fim, corrobora para ampliar a divulgação das responsabilidades deste profissional junto ao gerenciamento dos recursos materiais e humanos a fim de garantir uma assistência segura e qualificada ao paciente cirúrgico. Portanto, elaborou-se este estudo com o objetivo de relatar o potencial do trabalho do enfermeiro de centro cirúrgico para minimizar complicações pós-operatórias (OMS, 2009; SOUZA IP, 2018; EL-SAED A, et al., 2020; SOUZA CDM, et al., 2020; BADIA JM, et al., 2017; GOMES ET, et al., 2020).

\section{RELATO DE EXPERIÊNCIA}

Trata-se de um estudo descritivo, do tipo relato de experiência, construído por discentes e docentes de enfermagem a partir de atividades práticas com pacientes cirúrgicos. Desenvolvidas de março a junho de 2019 em um hospital filantrópico localizado na Fronteira Oeste do estado Rio Grande do Sul, Brasil. O referido hospital possui 199 leitos de internação, destes 16 situam-se no CC, no qual realizou-se a vivência prática.

Para a coleta de dados utilizaram-se os registros dos diários de campo e dos estudos de casos desenvolvidos pelas discentes e apreciados pelas docentes. Entre as informações, encontram-se os questionamentos realizados pelas docentes aos discentes, os dados da aplicação de instrumentos propiciados pelo componente curricular na avaliação da condição clínica dos pacientes cirúrgicos atendidos pelos discentes e docentes.

Os resultados deste relato de experiência estão abordados de modo qualitativo, sendo categorizados em: o potencial do enfermeiro para a segurança do paciente cirúrgico, o potencial do enfermeiro para 0 desenvolvimento da autonomia profissional e o potencial do enfermeiro para o trabalho colaborativo.

\section{O potencial do enfermeiro para a segurança do paciente cirúrgico}

No ambiente deste estudo, o $\mathrm{CC}$, ao longo das atividades práticas do curso de enfermagem pode-se vivenciar o potencial educativo na comunicação entre enfermeiros, docente, e destes com os discentes. $O$ enfermeiro do CC durante todo o período das atividades práticas respondeu aos questionamentos dos discentes, visto estarem sendo instigados pelo ambiente de prática e pelos docentes. A verbalização de conhecimentos ou dúvidas, ainda presentes quanto aos cuidados com pacientes cirúrgicos, estimulava a busca de respostas científicas para consolidar o conhecimento, fortalecer a habilidade e avaliar as possíveis atitudes para efetivar um cuidado integral e seguro ao paciente nos dias consecutivos à atividade prática.

Na perspectiva da segurança do paciente algumas ações foram aplicadas pelos discentes para ampliá-la, e de certo modo comunicar a equipe de enfermagem e demais profissionais do CC sua relevância para a qualidade do cuidado cirúrgico. Vinculava-se a identificação do paciente no avental e ao leito, com as respectivas informações: nome e a data de nascimento, visando estimular sua adoção pela equipe. $\mathrm{Na}$ prevenção de quedas teve-se o cuidado de elevar as grades dos leitos durante o transporte e mantê-las elevadas no tempo de duração do efeito anestésico. Para a garantia de um procedimento cirúrgico seguro buscou-se aplicar sob cuidados supervisionados a Lista de Verificação de Cirurgia Segura, mesmo não estando instituída no CC. 
Apesar da inserção prévia às atividades práticas, pelas docentes, e implementação da Escala de Avaliação de Risco para o Desenvolvimento de Lesões Decorrentes do Posicionamento Cirúrgico (ELPO) pelos discentes, a mesma não é aplicada pelos demais membros da equipe de enfermagem no cuidado ao paciente cirúrgico no cenário deste estudo. Destaca-se que nem mesmo outra escala era utilizada para esta avaliação. Não foi identificado conformidade para a higiene das mãos pelos profissionais de saúde no CC, conforme preconizado pelo Ministério da Saúde, pela Agência Nacional de Vigilância Sanitária e pela Rede Brasileira de Enfermagem e Segurança do Paciente.

Acrescessem ainda observação de não conformidade da técnica de escovação das mãos por todos os envolvidos no ato cirúrgico, inferindo lacunas no conhecimento essencial para o trabalho em CC. Esta higienização diferencia-se das demais porque tem-se a escovação associada ao uso de água e solução germicida. Todos os discentes puderam realizar a técnica de escovação das mãos e acompanhar a realização da técnica pelos demais discentes e profissionais, ao menos uma vez durante as atividades práticas sob supervisão da docente, visando refletir sobre a implementação da teoria na prática.

O acompanhamento do paciente desde seu ingresso no CC, do período pré-operatório imediato ao pósoperatório imediato, contribuiu para conhecer e provocar reflexões sobre o fluxograma de atendimento ao paciente e familiar. Além disso, forneceu subsídios para o aprofundamento e revisão farmacológica e na formação da enfermagem quanto ao efeito, dosagem, ação, interação medicamentosa e velocidade de infusão.

\section{O potencial para o desenvolvimento da autonomia profissional}

Os principais aspectos relativos à autonomia presenciados no CC durante as atividades práticas concernem às ações de gerenciamento pelo enfermeiro. Dentre estas, destaca-se o dimensionamento de pessoal por setor, a provisão e a previsão dos materiais para viabilizar o agendamento cirúrgico, atendimento das emergências, encaminhamentos de materiais aos laboratórios de análises clínica e/ou patológica, entre outras ações.

Apesar da proatividade do enfermeiro nesse setor, percebe-se a carência de um outro enfermeiro que possa responsabilizar-se pelas ações de assistência, cujas acabam, algumas vezes suprimidas ou subestimadas em sua realização. Acrescidos das demandas gerenciais e assistenciais no CC, este profissional deve atuar no atendimento das demandas do Centro Obstétrico (CO), que faz parte do espaço físico do CC neste campo de prática. Diante das demandas identifica-se mais do que a ampliação do quantitativo de enfermeiro, mas o potencial que a organização do trabalho fornece à autonomia do mesmo.

Para a maior seguridade e efetividade no cuidado cirúrgico, tem-se a possibilidade de incluir instrumentos de avaliação e de registro para as ações realizadas pela equipe multiprofissional, os quais subsidiarão as intervenções de enfermagem. Dentre as avaliações pode-se destacar a Lista de Cirurgia Segura, ELPO, Aldrete e Kroulik, entre outras. Tais instrumentos não utilizados em sua integridade na prática pelos profissionais, foram preenchidos pelos discentes em campo prático visando o aprofundamento teórico e seguridade documental, bem como promover a educação permanente dos profissionais envolvidos no cuidado nesta unidade.

A implementação de escalas validadas na prática de enfermagem perioperatória são capazes de assegurar uma assistência sistematizada ao paciente garantindo autonomia profissional e visibilidade da enfermagem.

\section{O potencial para o trabalho colaborativo}

São diversos desafios a serem apontados em todo e qualquer processo de trabalho desenvolvido em instituições filantrópicas sem fins lucrativos situadas em municípios do interior de um Estado, como na realidade do presente relato. Contraditoriamente estes desafios podem configurar-se em potencial para o trabalho colaborativo, isto porque tem-se menos profissionais e estes convivem por longos períodos diariamente. Logo, esperava-se que os ruídos e conflitos comunicacionais se apresentassem com menor incidência. 
A complexidade do trabalho do enfermeiro no CC destacou-se no presente estudo, especialmente no momento de busca por soluções rápidas e pontuais para assegurar a continuidade da assistência ao paciente cirúrgico. Fica notório que o uso da comunicação efetiva com todos os profissionais que circulam no ambiente foi primordial para o sucesso do trabalho, demonstrando que o enfermeiro é o meio existente para a tomada de decisão diante de situações emblemáticas ou da ocorrência de eventos adversos.

A conjugação da comunicação verbal e não verbal entre profissionais de enfermagem, médicos, farmacêuticos e pacientes possibilitou um real gerenciamento dos cuidados na busca de soluções rápidas e pontuais. A comunicação ao paciente resultante dessa conjugação de modos comunicacionais interprofissionais minimizou o estresse e ansiedade dos mesmos no CC. Enquanto que a comunicação com os familiares deu-se, na maioria das vezes, exclusivamente pela equipe médica.

\section{DISCUSSÃO}

O trabalho do enfermeiro visto pela holística deste relato, vai além de gerenciar a equipe de enfermagem e o CC, pois perpassa pelas múltiplas tarefas desse profissional, uma vez que, nesse ambiente tem-se a importância de acompanhar a transição do cuidado. A transição do cuidado no CC contempla tecnologias leve, leve-dura e dura as quais devem ser implementadas na articulação do cuidado assistencial e gerenciamento de pessoal. O potencial de atuação do enfermeiro reforça a autonomia e visibilidade profissional e seguridade na assistência e gerência do cuidado no CC (MARTINS FZ e DALL'AGNOL CM, 2016).

$\mathrm{Na}$ ótica do trabalho colaborativo, a atuação efetiva entre as equipes de enfermagem e demais membros devem incluir conhecimento, atitudes e a habilidade em gerenciar estratégias as quais promovam uma comunicação aberta com o respeito e a tomada de decisão compartilhada. Contudo, para ser efetivada na sua integridade torna-se essencial um processo sistematizado com divisão de tarefas objetivando os melhores resultados ao paciente (CHEEVER KH, 2016).

A oportunidade dos discentes de verbalizar conhecimentos ou inseguranças aos docentes ou à equipe de enfermagem demonstrou que o diálogo interprofissional, quando sustentado por conhecimento científico, fomenta a autonomia e o poder de decisão ao enfermeiro. Isto posto, a autonomia traduzida no uso da comunicação efetiva para a tomada de decisão produz dados e informações em prol da segurança do paciente e da qualidade no CC. Ademais, a habilidade comunicacional permite influenciar mudança cultural nos profissionais, permitindo melhores indicadores de qualidade à saúde (REBRAENSP, 2013; CARDOSO LS, et al., 2016).

A utilização de instrumentos na prática assegura o potencial do trabalho do enfermeiro para a segurança ao paciente, avaliação de indicadores de qualidade e continuidade no processo de trabalho colaborativo. No contexto de CC, destaca-se a Lista de Verificação de Cirurgia Segura que ao ser aplicada pelos profissionais nos três momentos do período operatório é capaz de reduzir incidentes, consequências à saúde e mortes cirúrgicas (OMS, 2009).

A implementação de ferramentas assistenciais na prática clínica, como a ELPO, prevê cuidados nas diferentes etapas cirúrgicas minimizando potenciais complicações pós-operatórias Destarte, a utilização conjunta dos instrumentos na prática por diferentes profissionais fortalece o trabalho colaborativo e incorpora esforços para a qualificação profissional por meio da educação permanente e continuada (LOPES et al, 2016; REEVES S, 2016).

A possibilidade de desenvolver a escovação das mãos pelos discentes é capaz de desenvolver habilidade, aperfeiçoar o conhecimento ao avaliar a técnica desenvolvida pelos profissionais e demais discentes, bem como refletir sobre os potenciais desfechos clínicos aos pacientes mediante a não conformidade da técnica. Outra possibilidade de desenvolvimento acadêmico é o acompanhamento do paciente no período pré, intra e pós-operatório os quais possibilitam aprofundamento sobre o efeito dos medicamentos, sejam anestésicos ou não, no cuidado de enfermagem. Tais oportunidades revelam o potencial do trabalho do enfermeiro e da importância do conhecimento científico na prática clínica. 
O quantitativo de profissionais atuantes no CC infere que a comunicação interprofissional seria um dos pontos positivos na continuidade do cuidado, no entanto, a identificação de situações específicas oportunizam o aprendizado no que se refere a importância da comunicação verbal e escrita. A comunicação seja oral ou escrita, deve ocorrer de forma inequívoca para que haja compreensão da equipe, pois uma interação deficiente pode causar danos e pôr em risco a saúde do paciente. Além disso, manter uma comunicação de qualidade contribui para tranquilizar e trazer maior vínculo e confiança entre profissional e paciente durante qualquer fase operatória (CAMARGO CP, et al., 2018).

A vivência dos discentes apontam o potencial do enfermeiro cirúrgico e o potencial para minimizar complicações no pós-operatório. A presença de docentes em campo prático no $\mathrm{CC}$ foi capaz de instigar conhecimentos e incertezas, as quais promovem o aprofundamento teórico e articulação teórico-prático. A utilização de instrumentos reforça que são ferramentas essenciais para garantir a qualidade da assistência prestada, bem como gerenciamento do serviço. As não conformidades na utilização de instrumentos ou técnicas de cuidado pelos profissionais demonstram fragilidades que devem ser reorganizadas para garantir a segurança do paciente, desenvolvimento da autonomia profissional e trabalho colaborativo.

\section{REFERÊNCIAS}

1. BADIA JM, et al. Impacto da infecção do sítio cirúrgico nos custos de saúde e nos resultados dos pacientes: uma revisão sistemática em seis países europeus. Journal of Hospital Infection, 2017; 96(1): 1-15.

2. CAMARGO CP, et al. Comunicação Terapêutica entre Paciente e Enfermagem no Período Perioperatório. Revista Multidisciplinar de Estudos Científicos em Saúde, 2018; 3(5): 38-42

3. CARDOSO LS, et al. The think of nursing in hospital urgency and emergency service. Revista de Enfermagem UFPE on line, 2016; 10: 4524-31.

4. CARVALHO R, BIANCHI ERF. Enfermagem em centro cirúrgico e recuperação. 2a Ed. São Paulo: Manole, 2016; 405p.

5. CONSELHO FEDERAL DE ENFERMAGEM. Resolução ํㅜ 564/2017. Aprova o novo Código de Ética dos Profissionais de Enfermagem. Diário Oficial da União: Brasília, DF, 2017.

6. DIEGO LAS, et al. Construção de uma ferramenta para medir percepções sobre o uso do Programa de Checklist de Cirurgia Segura da Organização Mundial da Saúde. Revista Brasileira de Anestesiologia, 2016; 66(4): $351-355$.

7. EL-SAED A, et al. High contribution and impact of resistant gram negative pathogens causing surgical site infections at a multi-hospital healthcare system in Saudi Arabia, 2007-2016. BioMed Central infectious diseases, 2020; 20(1).

8. REDE BRASILEIRA DE ENFERMAGEM E SEGURANÇA DO PACIENTE (REBRAENSP). Rede Brasileira de Enfermagem e Segurança do Paciente Estratégias para a segurança do paciente: manual para profissionais da saúde. Porto Alegre: EDIPUCRS, 2013. $132 \mathrm{p}$.

9. GOMES ET, et al. Can nursing actions prevent surgical wound dehiscence?. Rev SOBECC, 2020; 25(2): $114-119$.

10. LOPES CMM, et al. Escala de avaliação de risco para lesões decorrentes do posicionamento cirúrgico. Revista LatinoAmericana de Enfermagem. 2016; 24:e2704.

11. MACHADO MAG. A objeção de consciência no exercício da medicina: conflitos entre o dever ético-profissional e a autonomia de vontade do paciente. Dissertação (Mestrado em Ciência Jurídica) - Universidade do Vale do Itajaí, Itajaí, 2016; 139p.

12. MARTINS FZ, DALL'AGNOLB CM. Centro cirúrgico: desafios e estratégias do enfermeiro nas atividades gerenciais. Revista Gaúcha de Enfermagem, 2016; 37(4):e56945.

13. BRASIL. Agência Nacional de Vigilância Sanitária - ANVISA. Medidas de Prevenção de Infecção Relacionada à Assistência à Saúde, Brasília. 2017.

14. BRASIL. Organização Mundial de Saúde - OMS. Orientações da OMS para a Cirurgia Segura 2009 - Cirurgia Segura Salva Vidas, Brasília. 2009.

15. REEVES S. Por que precisamos de educação interprofissional para melhorar a prestação de cuidados seguros e eficazes. Interface (Botucatu), 2016; 20(56): 185-197.

16. SOBRAL GAS, et al. Atribuições do enfermeiro no centro cirúrgico. Enfermagem Brasil, 2019; 18(4): 603-609.

17. SOUZA CDM, et al. A importância da equipe de enfermagem na recuperação pós-anestésica. Faculdade Sant'Ana em Revista, 2020; 4(1): 4-13.

18. SOUZA IP. Análise da taxa de infecção de sítio cirúrgico no pós-operatório de cirurgia cardíaca. Trabalho de conclusão de curso (Graduação de enfermagem) - Faculdade de Medicina da Universidade Federal de Uberlândia, Uberlândia, 2018; 57p.

19. ZAMPER RPC, et al. O papel da tromboelastometria na avaliação e no tratamento da coagulopatia em pacientes submetidos ao transplante hepático. Einstein (São Paulo), 2017; 15(2): 243-246.

20. CHEEVER KH. Brunner e Suddarth: tratado de enfermagem médico-cirúrgica, volumes 1 e 2. 13 ed. Rio de Janeiro: Guanabara Koogan, 2016; 2175p. 